\title{
EPILEPSY
}

\section{Shining a light on seizure control-optogenetic approach shows promise for treatment and prevention of epilepsies}

Effective treatments for patients with epilepsy are lacking. Current antiepileptic therapies are often unsuccessful, with many patients failing to respond to drugs, and surgical treatment being feasible in only a minority of individuals. The 'holy grail' of epilepsy therapy is a treatment that targets only the brain regions involved in seizure generation and thus has no adverse effects on patient behaviour. According to two recent studies, optogenetics-the combined use of genetic and optical methods to control well-defined processes in a specific cell population-could provide the first step towards this goal.

In both studies, the researchers utilized viral vector-based gene therapy to transfect neurons of interest with halorhodopsin-a light-activated chloride pump from the bacterium Natronomonas pharaonis. In neurons, activation of the pump with yellow light enables transport of chloride ions into the cell, causing hyperpolarization of the membrane and a decrease in neuronal excitability. Optogenetic targeting of the neuronal populations that initiate epileptiform activity could, therefore, enable blockade of seizure generation.

In one of the studies, published in Science Translational Medicine, a team led by Dimitri Kullmann assessed the efficacy of optogenetic control of seizures in a tetanus toxin-induced rat model of epilepsy. "We decided to focus on focal neocortical epilepsy because it is difficult to treat," says Kullmann.

Halorhodopsin was transfected into pyramidal cells in the cortex at the same time as tetanus toxin administration. 1 week later, the animals were exposed to laser light in $20 \mathrm{~s}$ cycles via a cannula that was inserted into the cortex. After exposure to light, seizures were effectively blocked in the animals, as monitored using a novel wireless EEG transmitter technology. "We were able to suppress seizures acutely with only a small number of neurons expressing halorhodopsin," says
Kullmann. Notably, the animals displayed no signs of behavioural abnormalities.

Using the same model, but replacing halorhodopsin with $\mathrm{K}_{\mathrm{v}} 1.1$ (a mammalian potassium channel), Wykes et al. showed that continuous inhibition of action potential generation provided long-term protection from seizures. Furthermore, overexpression of $\mathrm{K}_{\mathrm{v}} 1.1$ in rats with established epilepsy had a curative effect.

Kullmann points out that refinement of optogenetic technologies is needed before this approach can be translated to clinical practice. "Nevertheless, we are pursuing these studies and, in the next few months, we are planning to implant animals with closed-loop devices that detect seizures and deliver light," he says.

The capacity of closed-loop optogenetic systems to control poststroke seizures was highlighted in another study by Jeanne Paz, John Huguenard and colleagues, published in Nature Neuroscience.

Paz et al. used an established photothrombotic model of focal cortical stroke, in which epilepsy occurs around 1 month after stroke, to assess which regions of the brain are involved in poststroke seizures. They discovered that this form of epilepsy was associated with damage to the thalamus-a structure that is remote from, but connected to, the injured cortex - and with hyperexcitablity of thalamocortical cells, suggesting that the thalamus is critical in poststroke seizure generation.

To test this theory, the researchers transfected neurons in the affected portion of the thalamus with halorhodopsin, and inserted a fibre optic cable to enable delivery of light. Electrodes were also implanted to record neural activity. "We connected all these devices to a multimodal electrical-optical interconnect, which enabled us to record epileptic activity and deliver light to the thalamus in awake, freely behaving animals, all in real time," explains Huguenard. Using a simple line-length

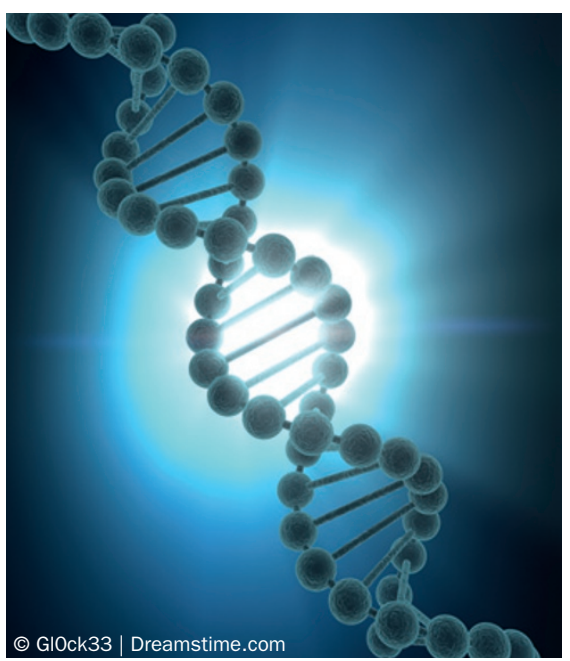

calculation, initiation of seizures could be reliably and specifically detected, and enabled delivery of light to the thalamus within $2 \mathrm{~s}$ of seizure onset.

Light-driven inhibition of neuronal activity in the thalamus was sufficient to interrupt seizure generation, with early abortion of epileptogenic activity in this region preventing its spread throughout the thalamocortical network. "This was the first demonstration of a real-time optogenetic approach to effectively stop seizures," says Huguenard.

Huguenard acknowledges that larger studies are needed to confirm the specificity and efficacy of the closed-loop optogenetic system for seizure control and to show that it does not affect normal behaviour, but he notes that the findings have strong implications for patients with epilepsy "if the twin challenges of gene therapy and light delivery can be met."

\section{Katy Malpass}

Original article Paz, J. T. et al. Closed-loop optogenetic control of thalamus as a tool for interrupting seizures after cortical injury. Nat. Neurosci. doi:10.1038/ nn.3269 | Wykes, D. C. et al. Optogenetic and potassium channel gene therapy in a rodent model of focal neocortical epilepsy. Sci. Transl. Med. doi:10.1126/ scitranslmed.3004190 